\title{
TRADICIÓN ORAL Y TRANSMISIÓN DE SABERES ANCESTRALES DESDE LAS INFANCIAS
}

Moreno-López, Nidia Milena; Sánchez-Torres, Antonio Isidro; Pérez-Raigoso, Andrea Del Pilar; AlfonsoSolano, Jorge Neftaly

TRADICIÓN ORAL Y TRANSMISIÓN DE SABERES ANCESTRALES DESDE LAS INFANCIAS

PANORAMA, vol. 14, núm. 26, 2020

Politécnico Grancolombiano, Colombia

Disponible en: http://www.redalyc.org/articulo.oa?id=343963784011

DOI: https://doi.org/10.15765/pnrm.v14i26.1489

Esta obra está bajo una Licencia Creative Commons Atribución-NoComercial-SinDerivar 4.0 Internacional. 
PANORAMA, vol. 14, núm. 26, 2020

Politécnico Grancolombiano, Colombia

Recepción: 13 Noviembre 2019 Aprobación: 05 Febrero 2020

DOI: https://doi.org/10.15765/ pnrm.v14i26.1489

Redalyc: http://www.redalyc.org/ articulo.oa? $\mathrm{id}=343963784011$
Artículos de investigación científica y tecnológica

\section{TRADICIÓN ORAL Y TRANSMISIÓN DE SABERES ANCESTRALES DESDE LAS INFANCIAS}

\author{
ORAL TRADITION AND TRANSMISSION OF \\ ANCESTRAL KNOWLEDGE FROM THE INFANTS \\ TRADIÇÃO ORAL E TRANSMISSÃO DO \\ CONHECIMENTO ANCESTRAL DOS INFANTIS
}

Nidia Milena Moreno-López nidia.morneo@unad.edu.co Universidad Nacional Abierta y a Distancia, UNAD., Colombia Antonio Isidro Sánchez-Torres antonio.sanchez@unad.edu.co Universidad Nacional Abierta y a Distancia, UNAD., Colombia Andrea Del Pilar Pérez-Raigoso andrea.perez@unad.edu.co Universidad Nacional Abierta y a Distancia, UNAD., Colombia Jorge Neftaly Alfonso-Solano jorge.alfonso880607@gmail.com Universidad Nacional Abierta y a Distancia, UNAD., Colombia

Resumen: En la actualidad existe un interés sobre la preservación de las culturas orales, teniendo en cuenta la multiciplidad de culturas ágrafas (Monsonyi, s.f). Es por ello que la presente investigación se centra en la importancia de la oralidad como transmisora de cultura en los primeros años. Para esto, valerse de las practicas pedagógicas y orientación educativa, desde perspectivas de inclusión, participación, diversidad y multiculturalidad, resulta una herramienta fundamental. En consecuencia, el realce, conservación y participación en usos y costumbres de la tradición oral de la primera infancia, apoyados en factores de liderazgo transformador y gestión asociativa (saber para saber), son una alternativa de innovación pedagógica que contribuye a que desde la infancia se instauren procesos cognitivos orientados a la preservación de los saberes culturales. Con este fin, se inicia desde el reconocimiento de las tradiciones en las regiones para así proponer y desarrollar acciones solidarias encaminadas a la manutención de los saberes populares, impactando desde la infancia en las regiones. El enfoque metodológico del presente estudio fue cualitativo, orientado a la comprensión de los fenómenos, indagando desde la perspectiva de los participantes en su contexto. Lo anterior en el marco del desarrollo de la Prestación del Servicio Social UnadistaSISSU, en articulación con el proyecto de investigación Tradición oral en las infancias: usos y costumbres. Los resultados alcanzados permitieron visibilizar la importancia del rescate, promoción y conservación de las diferentes tradiciones orales de nuestra multiculturalidad.

Palabras clave: Acción solidaria, lengua, pedagogía, infancia, tradición, oralidad, enseñanza, socialización.

Abstract: At present, there is an interest in the preservation of oral cultures, taking into account the multiculturality of literate cultures (Monsonyi, s.f). That is why the present investigation focuses on the importance of orality as a transmitter of culture in the first years. To do this, use pedagogical practices and educational guidance, from perspectives of inclusion, participation, diversity and multiculturalism 
is a fundamental tool. Consequently, the enhancement, conservation and participation in the uses and customs of the oral tradition of early childhood, supported by factors of transformative leadership and associative management (knowledge to know) are an alternative pedagogical innovation that contributes to childhood establish cognitive processes oriented in the preservation of cultural knowledge. For which, it starts from the recognition of traditions in the regions to propose and develop solidarity actions aimed at maintaining popular knowledge impacting from childhood in the regions. The methodological approach of the present study was qualitative oriented in the understanding of the phenomena, inquiring from the perspective of the participants in their context. The foregoing in the framework of the development of the Unified Social Service Provider-SISSU in conjunction with the research project Oral tradition in infants: Uses and customs. The results achieved made it possible to make visible the importance of the rescue, promotion and conservation of the different oral traditions of our multiculturalism.

Keywords: Solidarity action, language, pedagogy, childhood, tradition, orality, teaching, socialization.

Resumo: Atualmente, há interesse na preservação das culturas orais, levando em conta a multiculturalidade das culturas letradas (Monsonyi, s.f). É por isso que a presente investigação enfoca a importância da oralidade como transmissora da cultura nos primeiros anos. Para isso, utilizar práticas pedagógicas e orientação educacional, a partir de perspectivas de inclusão, participação, diversidade e multiculturalismo é uma ferramenta fundamental. Consequentemente, o aprimoramento, a conservação e a participação nos usos e costumes da tradição oral da primeira infância, apoiados por fatores de liderança transformativa e de gestão associativa (conhecimento para saber), são uma inovação pedagógica alternativa que contribui para a infância. estabelecer processos cognitivos orientados na preservação do conhecimento cultural. Para isso, parte do reconhecimento das tradições das regiões para propor e desenvolver ações solidárias que visem à manutenção do conhecimento popular impactante desde a infância nas regiões. A abordagem metodológica do presente estudo foi qualitativa orientada na compreensão dos fenômenos, indagando da perspectiva dos participantes em seu contexto. O que precede no âmbito do desenvolvimento do Prestador do Serviço Social Unificado SISSU, em articulação com o projeto de pesquisa Tradição oral em infantes: usos e alfândega. Os resultados obtidos permitiram tornar visível a importância do resgate, promoção e conservação das diferentes tradições orais do nosso multiculturalismo

Palavras-chave: Ação solidária, linguagem, pedagogia, infância, tradição, oralidade, ensino, socialização.

\section{INTRODUCCIÓN}

La acción solidaria que abarca el estudio holístico de la tradición oral en la primera infancia radica en las políticas gubernamentales de inclusión, participación, diversidad, multiculturalidad y respeto por los derechos fundamentales del niño (Sarlé, Ivaldi, \& Hernández, 2014). Promueve estrategias para la conservación de usos y costumbres desde la estética de la palabra viva, mediante las tradiciones orales como creaciones literarias, en apuesta a los niños y niñas como futuro inmediato de nuestro entorno integrado de desarrollo (Toro, 2012). Impulsadas desde la lúdica y didáctica referencial y significativa en el proceso de enseñanza y aprendizaje en prácticas pedagógicas suministradas a la población vinculada.

Con el diagnóstico participativo como herramienta en la asesoría educativa (Castro \& Alarcón, 2007), se ha evidenciado la necesidad de solucionar la problemática de la pérdida de identidad cultural y tradición 
oral de los pueblos latinoamericanos. Sobresale la importancia de la investigación regional de los usos y costumbres de la oralidad de nuestra multiculturalidad en aportes simbólicos y escritos (Montero, 2004; DazaOrozco, 2019). De esta manera, optimizar las prácticas pedagógicas en el proceso de enseñanza y aprendizaje para el desarrollo sostenible de la región. Esto desde el reconocimiento y preservación de la tradición oral de nuestros pueblos para el aprovechamiento de sus saberes populares en posibles respuestas de sus necesidades.

La UNAD, en su Cátedra Región, ha establecido el diagnóstico solidario como recurso inicial de reconocimiento del contexto local inmediato del estudiante. Este recurso permite indagar en las características propias del contexto, ahonda en las necesidades locales identificadas desde la disciplina del estudiante, en el marco del cumplimiento de los Objetivos de Desarrollo Sostenible en su región. De esta forma se ha identificado la importancia de resarcir las expresiones de tradición oral que acompañan a la primera infancia. Para ello se planifican y desarrollan acciones solidarias como aporte de la UNAD, en el marco de la interacción comunitaria y reconocimiento recíproco con las comunidades (Moreno, en prensa).

Los estudiantes de Licenciatura en Pedagogía infantil de la UNAD se hacen partícipes activos en el reconocimiento y accionar solidario como líderes transformadores mediante la Prestación del Servicio Social Unadista. Lo anterior apoyado desde el estudio de la teoría de la acción comunicativa de Garrido (2011), el desarrollo del diagnóstico solidario y la comprensión de la acción solidaria. Lo anterior aporta con evidencia empírica en el conocimiento de la naturaleza de la acción solidaria como elemento fundamental en el desarrollo del liderazgo transformador y la gestión asociativa, para que desde el saber para saber hacer se promueva la interacción y el diálogo con los diferentes líderes comunitarios, y aporte a la generación de soluciones específicas en referencia a problemáticas identificadas desde los diferentes proyectos de interacción social implementados (Moreno, 2018).

\section{Tradición oral en la primera infancia}

Las expresiones culturales de Colombia son manifestaciones del patrimonio cultural de cada lugar, y permiten el reconocimiento de la diferencia, de las tradiciones y la valoración de la tolerancia como elemento fundamental para una sana convivencia, en el marco de la diversidad y la multiculturalidad que exigen valores de respeto, solidaridad y consideración con aquello que es diferente y valioso desde su esencia histórica y cultural. Cada una de dichas expresiones ha constituido diferentes tipos de tradición oral, que hacen referencia a los conocimientos transmitidos de forma verbal de una generación a otra y que generalmente se da en el contexto familiar y comunitario, de modo que se favorezca la convivencia socializadora como ejemplo de integración de las infancias, desde las pautas culturales de la sociedad y desde el desarrollo comunitario y social de los diferentes grupos. Se reconoce 
que dichas prácticas aportan al desarrollo del pensamiento creativo y crítico como base para la consolidación de valores culturales, morales y éticos (Ramírez, 2009), lo que brinda autorreconocimiento y, por tanto, identidad.

Así, la importancia de la tradición oral desde perspectivas socioculturales, artísticas y de lenguaje autóctono ha estado principalmente orientada en caracterizar a los pueblos latinoamericanos. Sin embargo, en la actualidad, la incidencia de identidades ajenas a nuestras realidades aboca un estudio concienzudo desde la perspectiva de investigación y trabajo de campo (Rebollo, 2012), pues es evidente que lamentablemente en los pueblos latinoamericanos se viene presentando la pérdida de costumbres y tradiciones ancestrales, lo que lleva a que el patrimonio cultural se encuentre en proceso de extinción (Martínez, 2016); fenómeno que debe cuestionar nuestro quehacer desde el rol que cada uno asuma en su cotidianidad.

Por lo anterior, se hace necesario ahondar en el valor de las prácticas de tradición oral de los pueblos como eje fundamental de la cultura para la sostenibilidad y el desarrollo de las culturas y, por ende, de las regiones. En ese sentido, son varias las ventajas que trae consigo el ejercicio solidario del rescate de la tradición oral y que se relacionan con la importancia que tienen como elemento que fortalece el vínculo familiar que se transmite de generación en generación, la promoción del amor por la cultura como un sentimiento importante que se forja desde temprana edad y la interacción social desde la acción comunicativa en la construcción de nuevos conocimientos a partir de la sabiduría ancestral.

\section{Visualización de leyendas y mitos de la región}

Tomando como referente la didáctica de la lengua y la literatura, se pretende defender el enfoque comunicativo-funcional como agente protector de los procesos de enseñanza y de aprendizaje en las comunidades particulares, basados en la construcción conjunta de lo que los hechos lingüístico-comunicativos significan para todas las personas presentes en la educación (López \& Encabo, 2001a). De nada sirve reproducir las estructuras lingüísticas ya dadas, cuando lo que se provoca es el favorecimiento de la progresiva pérdida de la identidad lingüística. De ahí la relevancia de retomar y transmitir de manera permanente las leyendas, los mitos y todas aquellas manifestaciones de la tradición oral que representan para las comunidades ancestrales identidad, técnicas, creencias y saberes que constituyen un factor de vínculo y afecto entre todos los miembros de las comunidades.

\section{Lectura y comprensión de cuentos}

Hablar del género narrativo dentro de un contexto educativo supone que sea encasillado indistintamente en el género de la literatura infantil y juvenil; género que se caracteriza por unas figuras literarias particulares 
que tienen como foco edades específicas o está dirigida a personas que se hallan en edad evolutiva, es decir, entre los 4 y los 16 (Nobile, 1992). Esta podría ser la franja de edad en la que la narración sería susceptible de tener un valor didáctico que repercutiese en la formación de las personas. En acuerdo con Sánchez (1995a), la literatura infantil y juvenil posee un ideal propedéutico que está cimentado en la construcción del yo, en y por el discurso, lo que indica que, por medio de esta, tratamos de aproximarnos a la noción del ideal lingüístico-comunicativo, que mediante la comunicación literaria trata de iniciar a las nuevas generaciones en el diálogo cultural establecido en cualquier tipo de sociedad (Colomer, 1999).

\section{METODOLOGÍA}

El enfoque metodológico del presente estudio fue cualitativo orientado en la comprensión de los fenómenos, indagando desde la perspectiva de los participantes en su contexto. Lo anterior en el marco del desarrollo de la prestación del Servicio Social Unadista-SISSU, en articulación con el proyecto de investigación Tradición oral en las infancias: usos y costumbres. El estudio se estableció mediante el desarrollo de tres fases que se realizan en la comunidad con la que se interactúa: diagnóstico solidario, plan de acción solidaria y desarrollo de la acción solidaria.

\section{Fase 1. Diagnóstico solidario}

El prestador del Servicio Social Unadista realizó un reconocimiento teórico y una observación sobre las expresiones de tradición oral de su región que acompañan a la primera infancia. Este proceso de reconocimiento se orientó en la identificación de las expresiones orales relacionadas con los saberes tradicionales de su región (arrullo, canto, copla, cuento, retahíla, dichos, etc.), mediante diferentes fuentes, tanto primarias como secundarias. Esto con el fin de seleccionar alguna de las expresiones de la tradición oral de las regiones, y comprender el significado e importancia de los saberes regionales y la tradición oral como elementos del desarrollo integral de los niños y las niñas.

\section{Fase 2. Plan de acción}

Reconociendo las particularidades de la región y las características de las comunidades, producto de la fase inicial, se estructuró un plan de acción solidaria, dirigido a los niños y las niñas para promover el uso y práctica de las tradiciones orales seleccionadas mediante el diseño de un recurso pedagógico lúdico-didáctico. 


\section{Fase 3. Acciones solidarias}

Esta fase responde a la implementación de las acciones solidarias propuestas: las acciones solidarias son el resultado del pensamiento crítico y social de cada uno de los estudiantes, en correspondencia con las características de sus regiones a la luz de las temáticas propuestas en los proyectos solidarios. Para ello se convocó a la comunidad, particularmente a un grupo de niños y niñas, acompañados de sus madres, con quienes se socializó y desarrolló la acción solidaria planificada.

\section{INSTRUMENTOS}

En el proceso de observación e identificación de las tradiciones orales se realizó mediante diarios de campo y entrevistas no estructuradas dirigidas a la población de la que hace parte el estudiante servidor social.

\section{Población participante}

10 participantes con edades comprendidas entre 7 y 10 años, pertenecientes al corregimiento de Santa Cruz, departamento de Nariño, Colombia. 23 participantes con edades comprendidas entre 5 y 23 años. pertenecientes al barrio La Rosa, comuna 5, ciudad de San Juan de Pasto, departamento de Nariño, Colombia. 14 niños y niñas con un promedio de edad de 3 a 7 años, pertenecientes al hogar comunitario "Rositas", del municipio de Ipiales, vereda del corregimiento de Las Lajas.

\section{RESULTADOS}

A continuación se presentan los resultados obtenidos en tres acciones solidarias realizadas en el departamento de Nariño, en Colombia. Se inició con la recolección de información sobre tradiciones orales orientadas en las infancias. Posteriormente se definieron las tradiciones orales a promover: mitos y leyendas típicas de la comunidad. Finalmente, y mediante los procesos de enseñanza y aprendizaje de la comunidad en cuestión, se planificaron y desarrollaron las acciones solidarias.

\begin{tabular}{|c|c|c|c|}
\hline $\begin{array}{l}\text { Ubicación } \\
\text { geográfica }\end{array}$ & Diagnostico solidario y Plan de acción & $\begin{array}{l}\text { Acción solidaria en } \\
\text { tradición oral }\end{array}$ & Beneficiarios \\
\hline $\begin{array}{l}\text { Corregimiento } \\
\text { de Santa Cruz, } \\
\text { departamento } \\
\text { de Nariño, } \\
\text { Colombia. }\end{array}$ & $\begin{array}{l}\text { Se realizó en el corregimiento de Santa Cruz, } \\
\text { municipio de Policarpa, Nariño. Se encontró } \\
\text { receptividad por parte de la comunidad, pese } \\
\text { al contexto de violencia por conflicto armado } \\
\text { del que han sido víctimas. El reconocimiento } \\
\text { permitió establecer la necesidad de hacer } \\
\text { remembranza en algunos mitos y leyendas } \\
\text { propios de la región con contenidos } \\
\text { orientados al aprendizaje de valores desde la } \\
\text { infancia como la solidaridad, la convivencia, } \\
\text { el respeto y la ayuda a los demás. }\end{array}$ & $\begin{array}{l}\text { La acción solidaria planteada } \\
\text { se llevó a cabo el dia } 28 \text { de } \\
\text { noviembre de } 2017 \text {, en el } \\
\text { kiosco Vive digital del } \\
\text { corregimiento. Se orientó en } \\
\text { la lectura y comprensión } \\
\text { crítica y analítica de los } \\
\text { contenidos de cuentos y } \\
\text { leyendas propias de la } \\
\text { región. }\end{array}$ & $\begin{array}{l}\text { Los beneficiarios directos de } \\
\text { esta acción fueron los } 10 \\
\text { participantes, que } \\
\text { comprenden edades de } 7 \text { a } 10 \\
\text { años, quienes reflexionaron } \\
\text { sobre la importancia de } \\
\text { conocer y de replicar estas } \\
\text { tradiciones orales, por lo } \\
\text { relevantes para el aprendizaje } \\
\text { y práctica de algunos valores. }\end{array}$ \\
\hline
\end{tabular}

Tabla 1

Acción solidaria construyendo cultura con expresiones orales.

Los autores 


\begin{tabular}{|c|c|c|c|}
\hline $\begin{array}{l}\text { Ubicación } \\
\text { geográfica }\end{array}$ & $\begin{array}{c}\text { Diagnóstico solidario y Plan de } \\
\text { acción }\end{array}$ & Acción solidaria en tradición oral & Beneficiarios \\
\hline $\begin{array}{l}\text { Barrio La Rosa, } \\
\text { comuna 5, } \\
\text { ciudad de San } \\
\text { Juan de Pasto, } \\
\text { departamento } \\
\text { de Nariño, } \\
\text { Colombia. }\end{array}$ & $\begin{array}{l}\text { Esta comunidad está conformada por } \\
\text { personas de bajos recursos } \\
\text { socioeconómicos. El diagnóstico } \\
\text { solidario permitió establecer como } \\
\text { actividades prioritarias para la } \\
\text { promoción de la tradición oral } \\
\text { orientada en la primera infancia las } \\
\text { siguientes: } \\
\text { - Lectura y comprensión de mitos y } \\
\text { leyendas típicos de la región. Se } \\
\text { efectuaron tres tipos de lectura, se les } \\
\text { pidió a los niños que explicaran lo que } \\
\text { entendian. } \\
\text { - Visualización y análisis de bailes y } \\
\text { canciones nariñenses. Se mostraron } \\
\text { varios videos que incluian bailes de la } \\
\text { región. } \\
\text { - Creación de afiche con temáticas y } \\
\text { comprensión de cada niño y niña } \\
\text { sobre conceptos adquiridos } \\
\text { individualmente y sentimientos } \\
\text { generados. Realizaron carteleras, de } \\
\text { lo que, para ellos, trataban las } \\
\text { leyendas, bailes y culturas en general. }\end{array}$ & $\begin{array}{l}\text { La actividad se realizó el dia } 10 \text { de noviembre } \\
\text { en las instalaciones de la Fundación Mundo } \\
\text { Educativo, la cual dio inicio a las } 3: 00 \text { pm y } \\
\text { finalizó a las 5:00 pm, con un total de } 24 \\
\text { asistentes, } 21 \text { de ellos fueron participantes. Se } \\
\text { dividieron en dos grupos de acuerdo con su } \\
\text { edad. El primer grupo de } 5 \text { a } 10 \text { años, y el } \\
\text { segundo grupo de } 12 \text { a } 15 \text { años. Se inició con } \\
\text { la presentación del organizador y el objetivo } \\
\text { de la acción solidaria, seguido por la lectura } \\
\text { de un mito y una leyenda; seguido a esto se } \\
\text { contó la historia de los carnavales de blancos } \\
\text { y negros con imágenes alusivos a estos y se } \\
\text { finalizó con la presentación de un vídeo donde } \\
\text { se puede identificar el baile de la guaneña y } \\
\text { diferentes melodias de Nariño. Para evaluar la } \\
\text { acción solidaria y lograr el objetivo se realizó } \\
\text { un afiche creado por los propios niños, niñas y } \\
\text { adolescentes, con el fin de plasmar e } \\
\text { identificar lo que aprendieron de la tradición } \\
\text { oral nariñense. }\end{array}$ & $\begin{array}{l}\text { Los beneficiarios } \\
\text { presenciales de esta } \\
\text { acción fueron } 23 \\
\text { participantes } \\
\text { comprendidos entre } \\
\text { las edades de } 5 \text { a } 13 \\
\text { años, quienes } \\
\text { recibieron un } \\
\text { conocimiento directo, } \\
\text { pero los beneficiarios } \\
\text { serian incontables, } \\
\text { ya que, gracias a los } \\
\text { objetivos logrados en } \\
\text { la acción solidaria, } \\
\text { los niños y las niñas } \\
\text { serán los medios } \\
\text { para enseñar a } \\
\text { muchos otros sobre } \\
\text { la temática trabajada } \\
\text { en esta acción } \\
\text { solidaria. }\end{array}$ \\
\hline
\end{tabular}

Tabla 2.

Acción solidaria tradición oral en la comunidad infantil del barrio La Rosa. Los autores

\begin{tabular}{|c|c|c|c|}
\hline $\begin{array}{l}\text { Ubicación } \\
\text { geográfica }\end{array}$ & Diagnóstico solidario y Plan de acción & Acción solidaria en tradición oral & Beneficiarios \\
\hline $\begin{array}{l}\text { Hogar comunitario } \\
\text { "Rositas", del } \\
\text { municipio de } \\
\text { Ipiales, vereda del } \\
\text { corregimiento de } \\
\text { Las Lajas. }\end{array}$ & $\begin{array}{l}\text { Cumpliendo con el rescate de la identidad } \\
\text { cultural de la tradición oral de la cultura } \\
\text { pastos, enfocados en usos y costumbres } \\
\text { que se estaban perdiendo. Se encontró } \\
\text { que la comunidad está inmersa en un } \\
\text { entorno geográfico rural dependiente del } \\
\text { sector agrario y del turismo. En la región } \\
\text { se encuentran familias nucleares, con } \\
\text { indices importantes de violencia } \\
\text { intrafamiliar y bajo nivel socioeconómico. } \\
\text { Dentro del diagnóstico sobresale la } \\
\text { importancia de fortalecer la cultura de los } \\
\text { Pastos desde la tradición oral, expresada } \\
\text { mediante mitos, cuentos y leyendas. Lo } \\
\text { anterior se considera clave para atender } \\
\text { el problema de pérdida de identidad y } \\
\text { afianzar la multiculturalidad de los } \\
\text { pueblos. }\end{array}$ & $\begin{array}{l}\text { Las actividades concretamente realizadas } \\
\text { fueron: } \\
\text { - Mitos y leyendas de la cultura Pastos. Se } \\
\text { hizo lectura a cuentos propios de la región } \\
\text { como la Turumana y el Chutun } \\
\text { (Sistema Nacional de información Cultural, } \\
\text { Ministerio de Cultura). } \\
\text { - Posteriormente se realizaron logos, } \\
\text { emblemas e iconos de la cultura Pastos, } \\
\text { para fortalecer la identidad regional. Se } \\
\text { mostraron varios logos, para que los niños } \\
\text { los reconocieran como logos típicos de la } \\
\text { región } \\
\text { - Finalmente se realizaron prácticas } \\
\text { pedagógicas desde la lúdica y la didáctica } \\
\text { en referencia a tradiciones orales de los } \\
\text { ancestros de los pueblos Pastos. }\end{array}$ & $\begin{array}{l}\text { Los beneficiarios } \\
\text { presenciales de } \\
\text { esta acción } \\
\text { fueron } 14 \text { niños y } \\
\text { niñas con un } \\
\text { promedio de } \\
\text { edad de } 3 \text { a } 7 \\
\text { años. }\end{array}$ \\
\hline
\end{tabular}

Tabla 3.

Acción solidaria tradición oral "Leyendo y escribiendo mi vida voy construyendo”. Corregimiento de Las Lajas.

Los autores

\section{DISCUSIÓN Y CONCLUSIONES}

Las prácticas culturales de tradición oral se constituyen como acciones solidarias que favorecen la relación entre personas de diferentes edades para generar sentimientos de reconocimiento y aprecio frente a sus antepasados. De la misma manera, fortalecen el arraigo comunitario y, por ende, la identidad y pertenencia cultural, razón por la cual se hace necesario inculcarlo desde la infancia como "uno de los medios para asegurar la continuidad de un grupo social a través del fomento de la identidad cultural" (Ramírez 2009, p.23). 
Los resultados alcanzados permiten visibilizar la importancia del rescate, promoción y conservación de las diferentes tradiciones orales de nuestra multiculturalidad como zona sur de Colombia. Se concluye un impacto positivo que transversaliza el proceso de enseñanza y aprendizaje en el rescate, conservación y promulgación de las diferentes expresiones de tradición oral en nuestras comunidades. Lo anterior en pro del fortalecimiento cultural como elemento central de los Objetivos de Desarrollo Sostenible (ONU, 2017), y progreso de las regiones, desde la lúdica y didáctica.

Uno de los logros obtenidos de las acciones solidarias realizadas es la identificación de la apropiación de prácticas culturales desde los saberes ancestrales puestos en los contenidos de los cuentos, leyendas, mitos, canciones, bailes e historia originarios de la cultura nariñense. Saberes trasmitidos de generación en generación por la tradición oral y con un papel fundamental en la conducción social y política comunitaria como parte del control social.

Las acciones solidarias desarrolladas permitieron también evocar y dar a conocer algunos de los saberes regionales y manifestaciones de la tradición oral, como elementos clave para el desarrollo integral de los niños y niñas. Las actividades buscaron reforzar las estrategias de aprendizaje mediante la implementación de técnicas pedagógicas didácticas, con las cuales se abordó el tema por medio de los relatos orales, en pro de rescatar y conservar las tradiciones, la cultura, los conocimientos autóctonos de las regiones y la evolución de las comunidades.

En efecto, el recuperar estas prácticas de tradición oral que acompañan a niños y niñas en su primera infancia permite reestablecer y valorar las manifestaciones culturales de los antepasados, convirtiéndose en una estrategia educativa innovadora que beneficia a los infantes, ya que por medio de estos relatos interesantes van adquiriendo valores, enseñanzas y comportamientos que hacen que día a día la sociedad se transforme, evolucione y contribuya con el desarrollo sustentable de la región.

Este trabajo permitió visibilizar que mediante la implementación de acciones solidarias se fortalecen los procesos de asociatividad, evidenciados en el empoderamiento, apoyo, acompañamiento y promoción del trabajo en equipo, por un bien en pro de una comunidad o región mediante la integración y la interacción con las personas que hacen parte de una comunidad. Igualmente, se logra el afianzamiento de las tradiciones orales por medio de los mitos y leyendas, enfocándose en rescatar las buenas costumbres como una guía para que los niños sigan con estas tradiciones en generaciones posteriores dentro de las mismas comunidades.

Finalmente, se considera la importancia que tiene para la formación de los profesionales el aprendizaje y realización de acciones solidarias que contribuyan al cambio positivo de la sociedad como factor imprescindible para la edificación de comunidades más prósperas y desarrolladas. Cuando los estudiantes manifiestan que "como seres humanos hacemos parte de una sociedad de la cual debemos interesarnos, querer y valorar"; "trabajar por las comunidades es aportar un granito de arena al mundo 
entero, a nuestra vida y a nuestras generaciones"; "este trabajo nos deja como enseñanza ser más solidarios, interesarnos por el bien común" (Prestadores del Servicio Social Unadista, 2017), se valida la prioridad que tiene el trabajo educativo desde las necesidades pedagógicas, culturales y sociales de los niños y las niñas, con el fin de que el cambio se construya desde la perspectiva de los más vulnerables, trabajando conjuntamente por el desarrollo sostenible del mundo que han de vivir.

\section{REFERENCIAS}

1. Castro, P., Alarcón, M., Cavieres, H., Contreras, P., Inzunza, J., Marimbio, J., Palma, E., \& Tapia, S. (2007). El diagnóstico participativo como herramienta metodológica en la asesoría educativa. REICE. Revista Iberoamericana sobre Calidad, Eficacia y Cambio en Educación, 5(5e), 163-171.

2. Daza-Orozco, C. (2019). Historia de la infancia en el cine colombiano (1a ed.). Politécnico Grancolombiano. http://alejandria.poligran.edu.co/han $\mathrm{dle} / 10823 / 1648$

3. Fajardo, R. (2006). Prácticas socializadoras en la cultura Wayuu. Frónesis, 13(1), 19-31. Recuperado de: http://www.scielo.org.ve/scielo.php?script $=$ sci_arttext\&pid=S1315-62682006000100003\&lng=es\&tlng=es.

4. López, A., \& Encabo, E. (2013). De mitos, leyendas y cuentos: necesidad didáctica del género narrativo. Contextos Educativos. Revista de Educación, [S.1.](4), 241-250. ISSN 1695-5714. Recuperado de: https://publicacion es.unirioja.es/ojs/index.php/contextos/article/view/495/459

5. Garrido, L. (2011). Habermas y la teoría de la acción comunicativa. . azón y Palabra, (75). Recuperado de: http://www.razonypalabra.org.mx/N/N7 5/ultimas/38_Garrido_M75.pdf

6. Montero, M. (2004). El fortalecimiento en la comunidad, sus dificultades y alcances. Psychosocial Intervention, 13(1), 5-19. Recuperado de: http://w ww.redalyc.org/articulo.oa?id=179817825001

7. Obando, A. (2011). La comuna cinco en Pasto. Recuperado de: arturobando.blogspot.com/2011/10/la-comuna-cinco-en-pasto.html

8. Prieto, R., \& Fabelo, R. (2009). Liderazgo y capital social: uso de redes como herramienta para el desarrollo sostenible. Telos, 11(1), 52-68.

9. Ramírez. A, Sánchez. J, \& García, A. (2004). El desarrollo sustentable: interpretación y análisis. Universidad La Salle. Revista del Centro de Investigación, 6(21), 55-56. Recuperado de: http://www.redalyc.org/artic ulo.oa?id=34202107

10. Rebollo, Ó. (2012). La transformación social urbana. La acción comunitaria en la ciudad globalizada. Gestión y Política Pública, 159-186. Recuperado de: http://www.redalyc.org/articulo.oa?id=13324931005

11. Toro, D. (2012). Estética de la palabra viva. Las tradiciones orales como creaciones literarias. Boletín de Antropologia Universidad de Antioquia, $26(43), 260-262$. 